\title{
Ampleness of two-sided tilting complexes
}

\author{
Hiroyuki Minamoto
}

March 7, 2009

\begin{abstract}
In this paper we define the notion of ampleness for two-sided tilting complexes over finite dimensional algebras and prove its basic properties.

We call a finite dimensional $k$-algebra $A$ of finite global dimension Fano if $\left(A^{*}[-d]\right)^{-1}$ is ample for some $d \geq 0$. For example geometric algebras in the sense of Bondal-Polishchuk are Fano. We give a characterization of representation type of a quiver from a noncommutative algebro-geometric view point, that is, a finite acyclic quiver has finite representation type if and only if its path algebra is fractional Calabi-Yau, and a finite acyclic quiver has infinite representation type if and only if its path algebra is Fano.
\end{abstract}

\section{Introduction}

Let $X$ be a nonsingular projective variety over a field $k$ and let $\omega_{X}$ be its canonical bundle. Then the functor $S_{X}:=-\otimes_{X}^{\mathbf{L}} \omega_{X}[\operatorname{dim} X]: D^{b}(\operatorname{coh} X) \longrightarrow D^{b}(\operatorname{coh} X)$ is the Serre functor ,i.e., $\operatorname{Hom}_{X}\left(\mathcal{G}^{*}, \mathcal{F}^{*}\right)^{*}$ is functorially isomorphic to $\operatorname{Hom}_{X}\left(\mathcal{F}, S_{X}(\mathcal{G})\right)$ for $\mathcal{F}, \mathcal{G} \in D^{b}(\operatorname{coh} X)$. By this fact, from a noncommutative (or categorical) algebro-geometric view point, one thinks of a triangulated category $\mathcal{T}$ as the derived category of coherent sheaves of some "space" $X$ and of the Serre functor $S_{\mathcal{T}}$ of $\mathcal{T}$ (if exists) as the derived tensor product of " $\operatorname{dim} X$ "-shifted "canonical bundle" $\omega_{X}$. From this view point, the notion of Calabi-Yau algebra ( and Calabi-Yau category ) is defined and studied extensively by many researchers.

In this paper we introduce the notion of ampleness for two-sided tilting complexes over finite dimensional $k$-algebras. Let $A$ be a finite dimensional $k$-algebra of finite global dimension.

Definition 0.1 (Definition 2.6). A two-sided tilting complex $\sigma$ over $A$ is called very ample if $\mathrm{H}^{i}(\sigma)=0$ for $i \geq 1$ and $\sigma^{n}$ is pure for $n \gg 0 . \sigma$ is called ample if $\sigma^{n}$ is pure for $n \gg 0$.

In Section 2, we justify this definition by using the theory of noncommutative projective schemes due to Artin-Zhang [AZ] and Polishchuk [Po]. In the theory of noncommutative projective schemes , for a graded coherent ring $R$ over $k$ we attach an imaginary geometric object $\operatorname{proj} R=(\operatorname{cohproj} R, \bar{R},(1))$. An abelian category cohproj $R$ is considered as the category of coherent sheaves on proj $R$. (See Section 1.) In Section 2 we show that the following facts hold. If $\sigma$ is a very ample tilting complex over $A$, then the tensor algebra $T:=T_{A}\left(\mathrm{H}^{0}(\sigma)\right)$ of $\mathrm{H}^{0}(\sigma)$ over $A$ is a graded connected coherent ring over $A$ and there is a $t$-structure $D^{\sigma}$ defined by $\sigma$ in $\operatorname{Perf} A$ and its heart $\mathcal{H}^{\sigma}$ is equivalent to $\operatorname{cohproj} T$. Moreover the following Theorem holds.

Theorem 0.2 (Theorem 2.8). Let $A$ be a finite dimensional $k$-algebra of finite global dimension and let $\sigma$ be a very ample two-sided tilting complex. Then there is a natural equivalence of triangulated categories

$$
D^{b}(\bmod -A) \stackrel{\sim}{\longrightarrow} D^{b}(\operatorname{cohproj} T) .
$$

where $T:=T_{A}\left(\mathrm{H}^{0}(\sigma)\right)$ is the tensor algebra of $\mathrm{H}^{0}(\sigma)$ over $A$. 
In $[\mathrm{Be}]$ Beilinson showed that $\mathbb{P}^{n}$ is derived equivalent to a finite dimensional $k$-algebra. This result has been generalized to other varieties. The above Theorem gives a partial converse.

A finite dimensional $k$-algebra $A$ of finite global dimension is called Fano if $\left(A^{*}[-d]\right)^{-1}$ is ample for some $d \geq 0$.

We give a characterization of representation type of a quiver from noncommutative algebrogeometric view point, that is, a finite acyclic quiver has finite representation type if and only if its path algebra is fractional Calabi-Yau, and a finite acyclic quiver has infinite representation type if and only if its path algebra is Fano.

In [Le] and [GL], geometric notions are introduced to study certain class of algebras. This paper develop a formal aspect of these works.

This paper is generalization of [Mi].

We organize the present paper as follows: in Section 1 we introduce some definitions and results on noncommutative projective schemes :in Section 2 we give the definition of ampleness of two-sided tilting complexes and prove its basic property : in Section 3 we show that some finite dimensional algebra studied before has ample or anti-ample "canonical bundle".

Notation and convention. Throughout this paper $k$ denotes a field. If there would be no confusion, we denote by the same symbol $T$ a two-sided tilting complex $T$ and the functor $-\otimes_{A}^{\mathbf{L}} T$ induced by $T$. For a $\operatorname{ring} A$ we denote by $\operatorname{Mod}-A(\operatorname{resp} . \bmod -A$ ) the abelian category of right $A$-modules (resp. the abelian category of finite right $A$-modules). For a $k$-vector space $M$, we denote by $M^{*}$ its $k$-dual vector space.

Acknowledgment. The author is grateful to I. Mori for his great help and comment. The author thank H. Asashiba, O. Iyama, and A. Takahashi for their comments and useful discussions on this paper. The author thank Y. Kimura for suggestive discussions.

\section{Preliminaries on Noncommutative Projective Schemes}

This section is a summary of the paper $[\mathrm{Po}]$ by A. Polishchuk. Although connected $\mathbb{Z}$-algebras are treated in $[\mathrm{Po}]$, we treat connected $\mathbb{N}$-graded algebras over some finite dimensional $k$-algebra $A$. One can see that the argument in $[\mathrm{Po}]$ is applied to our case.

Let $k$ be a field and let $R=R_{0} \oplus R_{1} \oplus R_{2} \oplus \cdots$ be a graded coherent ring over $k$. We assume that the degree zero part $R_{0}$ of $R$ is a finite dimensional algebra over $k$. Gr $R$ (resp. coh $R$ ) denotes the category of graded right $R$-modules (resp. finitely presented graded right $R$-modules). Tor $R$ (resp. tor $R$ ) denote the full subcategory of torsion modules (resp. modules finite dimensional over $k$ ). Note that Tor $R$ and tor $R$ are dense subcategories of $\operatorname{Gr} R$ and $\operatorname{coh} R$ respectively, hence the quotient categories QGr $R=\operatorname{Gr} R /$ Tor $R$ and cohproj $R=\operatorname{coh} R /$ tor $R$ are abelian categories.

For a graded right $R$-module $M=\oplus_{n \in \mathbb{Z}} M_{n}$, we denote by $M(1)$ the 1-degree shift of $M$. i.e., $M(1)_{n}=M_{n+1}$. The degree shift operator $(1): \operatorname{coh} R \longrightarrow \operatorname{coh} R$ induces the autoequivalence (1) on cohproj $R$. We denote by $\bar{R}$ the image in cohproj $R$ of the regular module $R_{R}$. The (coherent) noncommutative projective scheme proj $R$ associated to $R$ is the triple (cohproj $R, \bar{R},(1))$. The autoequivalence (1) is called the canonical polarization on proj $R$.

In noncommutative projective geometry, one thinks of cohproj $R$ as the category of coherent sheaves on a noncommutative projective scheme $\operatorname{proj} R$ associated to a graded ring $R$ ([AZ, Po]).

Let us consider a triple $(\mathcal{C}, \mathcal{O}, s)$ consisting of a $k$-linear abelian category $\mathcal{C}$ such that $\operatorname{dim}_{k}(\mathcal{F}, \mathcal{G})<$ $\infty$ for any $\mathcal{F}, \mathcal{G} \in \mathcal{C}$, an object $\mathcal{O} \in \mathcal{C}$ and an autoequivalence $s$ on $\mathcal{C}$. For $\mathcal{F} \in \mathcal{C}$, we define

$$
\Gamma_{*}(\mathcal{F})=\bigoplus_{n \geq 0} \operatorname{Hom}_{\mathcal{C}}(\mathcal{O}, \mathcal{F}(n))
$$


where $\mathcal{F}(n):=s^{n} \mathcal{F}$, and we set

$$
R=\Gamma_{*}(\mathcal{C}, \mathcal{O}, s)=\Gamma_{*}(\mathcal{O}) .
$$

Multiplication is defined as follows: If $x \in \operatorname{Hom}_{\mathcal{C}}(\mathcal{O}, \mathcal{F}(l)), b \in \operatorname{Hom}_{\mathcal{C}}(\mathcal{O}, \mathcal{O}(m))$ and $a \in$ $\operatorname{Hom}_{\mathcal{C}}(\mathcal{O}, \mathcal{O}(n))$, then

$$
x \cdot a=s^{n}(x) \circ a \quad \text { and } \quad a \cdot b=s^{m}(a) \circ b .
$$

With this law of composition, $\Gamma_{*}(\mathcal{F})$ becomes a graded right module over the graded algebra $R$ over $k$.

Definition 1.1 ([AZ, Section 4.2],[Po, Section 2]). Let $(\mathcal{C}, \mathcal{O}, s)$ be a triple as above. Then the pair $(\mathcal{O}, s)$ is called ample if the following conditions hold:

(1) For every object $\mathcal{F} \in \mathcal{C}$, there are positive integers $l_{1}, \ldots, l_{p}$ and an epimorphism $\oplus_{i=1}^{p} \mathcal{O}\left(-l_{i}\right) \longrightarrow$ $\mathcal{F}$.

(2) For every epimorphism $f: \mathcal{F} \longrightarrow \mathcal{G}$, there exists an integer $n_{0}$ such that for every $n \geq n_{0}$ the induced map $\operatorname{Hom}_{\mathcal{C}}(\mathcal{O}, \mathcal{F}(n)) \longrightarrow \operatorname{Hom}_{\mathcal{C}}(\mathcal{O}, \mathcal{G}(n))$ is surjective.

Let $\pi: \operatorname{Gr} R \longrightarrow$ QGr $R$ be the quotient functor. Set $\bar{\Gamma}_{*}=\pi \circ \Gamma_{*}$.

Theorem 1.2 ([Po, Theorem 2.4]). Let $(\mathcal{C}, \mathcal{O}, s)$ be a triple as above. If $(\mathcal{O}, s)$ is ample, then the graded ring $R=\Gamma_{*}(\mathcal{C}, \mathcal{O}, s)$ is coherent, $\Gamma_{*}(\mathcal{F})$ is finitely presented $R$-module for $\mathcal{F} \in \mathcal{C}$ and the functor $\bar{\Gamma}_{*}: \mathcal{C} \longrightarrow$ cohproj $R$ induces an equivalence of triples between $(\mathcal{C}, \mathcal{O}, s)$ and proj $R=$ (cohproj $R, \bar{R},(1))$, i.e.,

$$
\begin{aligned}
& \bar{\Gamma}_{*}: \mathcal{C} \stackrel{\sim}{\longrightarrow} \text { cohproj } R \text { is an equivalence of categories, } \\
& \bar{\Gamma}_{*}(\mathcal{O}) \cong \bar{R} \text {, and } \bar{\Gamma}_{*} \circ s=(1) \circ \bar{\Gamma}_{*} .
\end{aligned}
$$

\section{$2 \quad t$-structures defined by two-sided tilting complexes}

Let $X$ be a projective variety over $k$ and $\mathcal{L}$ be an ample line bundle on $X$. Let $\left(D^{\geq 0}, D^{\leq 0}\right)$ be the standard $t$-structure in $D^{b}(\operatorname{coh} X)$,i.e., $D^{\geq 0}$ (resp. $\left.D^{\leq 0}\right)$ is the full subcategory of $D^{b}(\operatorname{coh} X)$ with objects $\mathcal{F}$ such that $\mathrm{H}^{i}\left(\mathcal{F}^{\cdot}\right)=0$ for $i<0$ (resp. $i>0$ ). By Serre's vanishing theorem [Har, Propsition III.5.3], a complex $\mathcal{F} \in D^{b}(\operatorname{coh} X)$ belongs to $D^{\geq 0}\left(\right.$ resp. $\left.D^{\leq 0}\right)$ if and only if $\mathcal{F}$ satisfies the following condition:

$$
\begin{array}{rlr} 
& \mathbb{R} \operatorname{Hom}\left(\mathcal{O}_{X}, \mathcal{F} \otimes^{\mathbf{L}} \mathcal{L}^{n}\right) \in D^{\geq 0}(k \text {-vect }) & \text { for } n \gg 0 \\
\text { (resp. } & \mathbb{R} \operatorname{Hom}\left(\mathcal{O}_{X}, \mathcal{F} \otimes^{\mathbf{L}} \mathcal{L}^{n}\right) \in D^{\leq 0}(k \text {-vect }) . & \text { for } n \gg 0)
\end{array}
$$

Reversing this observation, to formulate ampleness in the study of derived categories, we define the following.

Definition 2.1. Let $A$ be a k-algebra and let $\sigma$ be a two-sided tilting complex over $A$. The full subcategory $D^{\sigma, \geq 0}\left(\right.$ resp. $\left.D^{\sigma, \leq 0}\right)$ of $D^{b}(\bmod -A)$ consists of objects $M$ which satisfy

$$
\begin{aligned}
& \sigma^{n} M \in D^{\geq 0}(\operatorname{Mod}-A) & \text { for } n \gg 0 \\
(\text { resp. } & \sigma^{n} M \in D^{\leq 0}(\operatorname{Mod}-A) & \text { for } n \gg 0) .
\end{aligned}
$$

We define $D^{\sigma}:=\left(D^{\sigma, \geq 0}, D^{\sigma, \leq 0}\right)$.

Since $\sigma^{n} M \simeq \mathbb{R} \operatorname{Hom}\left(A, \sigma^{n} M\right)$, we think of $A$ as the "structure sheaf" in Definition 2.1. 
Theorem 2.2. Let $A$ be a right Noetherian k-algebra of finite global dimension and let $\sigma$ be a twosided tilting complex over $A$. If $\mathrm{H}^{i}(\sigma)=0$ for $i \geq 1$, then $D^{\sigma}$ is a t-structure in $D^{b}(\bmod -A)$.

To prove Theorem 2.2 we need the following Lemma.

Lemma 2.3. Let $f: L \longrightarrow M$ be a morphism in $D^{-}\left(\operatorname{Mod}-A \otimes_{k} B^{\mathrm{op}}\right)$ and $N \in D^{-}\left(\operatorname{Mod}-C \otimes_{k} A^{\mathrm{op}}\right)$ for $k$-algebras $A, B, C$. If $\mathrm{H}^{i}(f)=0$ for any $i \in \mathbb{Z}$, then $\mathrm{H}^{i}\left(f \otimes_{A}^{\mathbf{L}} 1_{N}\right)=0$ for any $i \in \mathbb{Z}$.

The above Lemma is easily proved by the following Lemma taken from [Y, Lemma 2.1]. (See also [ML, Theorem XII. 12.2].)

Lemma 2.4. Let $M \in D^{-}\left(\operatorname{Mod}-A \otimes_{k} B^{\text {op }}\right)$ and $N \in D^{-}\left(\operatorname{Mod}-C \otimes_{k} A^{\text {op }}\right)$ for $k$-algebras $A, B, C$. Then there is a convergent Künneth spectral sequence

$$
\mathrm{E}_{2}^{p, q}=\bigoplus_{i+j=q} \mathrm{H}^{p}\left(\mathrm{H}^{i}(M) \otimes_{A}^{\mathbf{L}} \mathrm{H}^{j}(N)\right) \Longrightarrow \mathrm{H}^{p+q}\left(M \otimes_{A}^{\mathbf{L}} N\right)
$$

in Mod- $C \otimes_{k} B^{\mathrm{op}}$ which is functorial in $M$ and $N^{\cdot}$. If $i_{0} \geq \sup \left\{i \mid \mathrm{H}^{i}(M) \neq 0\right\}$ and $j_{0} \geq \sup \{j \mid$ $\left.\mathrm{H}^{j}(N) \neq 0\right\}$, then $\mathrm{H}^{i_{0}}(M) \otimes_{A} \mathrm{H}^{j_{0}}(N) \simeq \mathrm{H}^{i_{0}+j_{0}}\left(M \otimes_{A}^{\mathbf{L}} N\right)$.

The latter part of this Lemma will be used in the sequel.

Proof of Theorem 2.2. The only nontrivial part is the following statement:

For any $M \in D^{b}(\bmod -A)$ there is an exact triangle

$$
M^{\prime} \longrightarrow M \longrightarrow M^{\prime \prime} \stackrel{[1]}{\longrightarrow}
$$

in $D^{b}(\bmod -A)$ such that $M^{\prime} \in D^{\sigma, \leq 0}$ and $M^{\prime \prime} \in D^{\sigma, \geq 1}$.

Let $\left(D^{\leq 0}, D^{\geq 0}\right)$ be a standard $t$-structure in $D(\operatorname{Mod}-A)$ and let $\tau_{\leq 0}$ and $\tau_{\geq 1}$ be standard truncation functors. Let $N \in D^{b}(\bmod -A)$. Applying $\sigma$ to the canonical morphism $\tau_{\leq 0} N \longrightarrow N$, we get the morphism $\sigma\left(\tau_{\leq 0} N\right) \longrightarrow \sigma(N)$. Since $\sigma$ is the derived tensor $-\otimes_{A}^{\mathbf{L}} \sigma$ of the complex $\sigma$ such that $\mathrm{H}^{i}(\sigma)=0$ for $i \geq 1, \sigma\left(\tau_{\leq 0}(N)\right) \in D^{\leq 0}$. Therefore we get a morphism $\sigma\left(\tau_{\leq 0}(N)\right) \longrightarrow \tau_{\leq 0}(\sigma(N))$. Setting $N=\sigma^{n}(M)$ for $n \geq 0$, we get a morphism $\sigma\left(\tau_{\leq 0}\left(\sigma^{n} M\right)\right) \longrightarrow \tau_{\leq 0}\left(\sigma^{n+1} M\right)$. Applying $\sigma^{-(n+1)}$ to this morphism, we get a morphism $\phi_{n}: \sigma^{-n}\left(\tau_{\leq 0}\left(\sigma^{n} M\right)\right) \longrightarrow \sigma^{-(n+1)}\left(\tau_{\leq 0}\left(\sigma^{n+1} M\right)\right)$. Set $\tau_{\leq 0}^{\sigma, n}:=\sigma^{-n} \tau_{\leq 0} \sigma^{n}$ and $\tau_{\geq 1}^{\sigma, n}:=\sigma^{-n} \tau_{\geq 1} \sigma^{n}$. Applying $\sigma^{-n}$ to the exact triangle

$$
\tau_{\leq 0}\left(\sigma^{n} M\right) \longrightarrow \sigma^{n} M \longrightarrow \tau_{\geq 1}\left(\sigma^{n} M\right) \stackrel{[1]}{\longrightarrow},
$$

we get the following exact triangle

$$
\tau_{\leq 0}^{\sigma, n} M \stackrel{\alpha_{n}}{\longrightarrow} M \stackrel{\beta_{n}}{\longrightarrow} \tau_{\geq 1}^{\sigma, n}(M) \stackrel{[1]}{\longrightarrow} .
$$

We have the following commutative diagram:

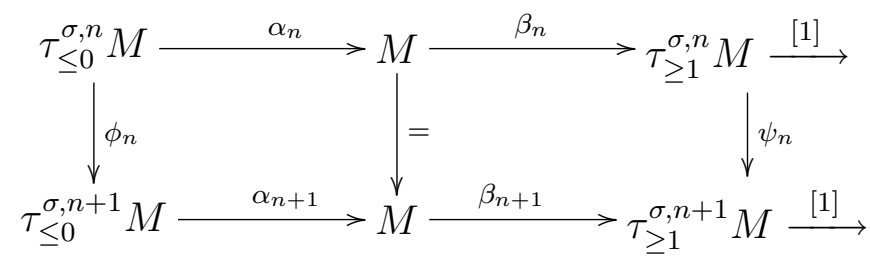

where $\psi_{n}$ is induced morphism. 
Let us consider the following cohomology long exact sequence of (2):

$$
\cdots \rightarrow \mathrm{H}^{i-1}\left(\tau_{\geq 1}^{\sigma, n} M\right) \stackrel{\partial^{i-1}}{\longrightarrow} \mathrm{H}^{i}\left(\tau_{\leq 0}^{\sigma, n} M\right) \stackrel{\mathrm{H}^{i}\left(\alpha_{n}\right)}{\longrightarrow} \mathrm{H}^{i}(M) \rightarrow \cdots
$$

where $\partial^{i-1}$ is the connecting morphism. Let $\delta: \tau_{\geq 1} \sigma^{n} M \longrightarrow \tau_{\leq 0} \sigma^{n} M[1]$ be the morphism obtained by rotating the exact triangle $(1)$. Then $\mathrm{H}^{i}(\delta)=0$ for any $i \in \mathbb{Z}$. Since $\partial^{i-1} \simeq \mathrm{H}^{i-1}\left(\delta \otimes_{A}^{\mathbf{L}} 1_{\sigma^{-n}}\right)$, $\partial^{i-1}=0$ by Lemma 2.3. Therefore $\mathrm{H}^{i}\left(\alpha_{n}\right)$ is injective. Hence $\mathrm{H}^{i}\left(\phi_{n}\right)$ is injective. We have the following system of injections

$$
\cdots \hookrightarrow \mathrm{H}^{i}\left(\tau_{\leq 0}^{\sigma, n} M\right) \hookrightarrow \mathrm{H}^{i}\left(\tau_{\leq 0}^{\sigma, n+1} M\right) \hookrightarrow \cdots \quad \mathrm{H}^{i}(M) .
$$

Since $\mathrm{H}^{i}(M)=0$ except for finitely many $i$ and $\mathrm{H}^{i}(M)$ is Noethrian for each $i$, there is a positive integer $n_{0}$ such that $\mathrm{H}^{i}\left(\phi_{n}\right)$ is isomorphism for $n \geq n_{0}$ and $i \in \mathbb{Z}$. Therefore $\phi_{n}$ and $\psi_{n}$ is quasiisomorphism for $n \geq n_{0}$. Thus if we set $M^{\prime}:=\tau_{\leq 0}^{\sigma, n_{0}} M$ and $M^{\prime \prime}:=\tau_{\geq 1}^{\sigma, n_{0}} M$, then $M^{\prime} \in D^{\rho, \leq 0}$ and $M^{\prime \prime} \in D^{\rho, \geq 1}$. This complete the proof of the Theorem.

Let $A$ be a finite dimensional $k$-algebra of finite global dimension and $\sigma$ be a two-sided tilting complex over $A$ such that $\mathrm{H}^{i}(\sigma)=0$ for $i \geq 1$. Then by Theorem $2.2, D^{\sigma}$ is $t$-structure in $D^{b}(\bmod -A)$. Let $\mathcal{H}^{\sigma}$ be the heart of the $t$-structure $D^{\sigma}$. Then $\sigma$ acts on $\mathcal{H}^{\sigma}$. Furthermore assume that $\sigma^{n}$ is pure in standard $t$-structure for each $n \gg 0$. Then $A \in \mathcal{H}^{\sigma}$ and the triple $\left(\mathcal{H}^{\sigma}, A, \sigma\right)$ satisfies the conditions in Section 1.

Lemma 2.5. With the assumptions above the pair $(A, \sigma)$ is ample in the sense of Definition 1.1 on the triple $\left(\mathcal{H}^{\sigma}, A, \sigma\right)$.

Proof. We check the conditions (1) and (2) of Definition 1.1.

First note that the cokernel of the morphism $f: M \longrightarrow N$ in the abelian category $\mathcal{H}^{\sigma}$ is $\tau_{\geq 0}^{\sigma}($ Cone $(f))$, where $\tau_{\geq 0}^{\sigma}: D^{b}(\bmod -A) \longrightarrow D^{\sigma, \geq 0}$ is the truncation functor of the $t$-structure $D^{\sigma}$ (See [GM, IV.4]). So $f$ is an epimorphism in $\mathcal{H}^{\sigma}$ if and only if Cone $(f) \in D^{\sigma, \leq-1}$.

(1) Let $M \in \mathcal{H}^{\sigma}$ and let $n \geq 0$ be an integer such that $\sigma^{n} M$ is pure. Let $P$ be a bounded complex of finite projective right $A$-modules which represents $\sigma^{n} M$. We may assume that $P^{i}=0$ for $i \geq 1$ and $P^{0} \cong A^{\oplus p}$ for some $p \in \mathbb{N}$. An isomorphism $A^{\oplus p} \stackrel{\sim}{\rightarrow} P^{0}$ induces a morphism $\phi: A^{\oplus p} \longrightarrow \sigma^{n} M$. Let $C$ be a cone of $\phi$. Then $C \in D^{\leq-1}$ and we have the following exact triangle

$$
\sigma^{-n} A^{\oplus p} \stackrel{\sigma^{-n} \phi}{\longrightarrow} M \longrightarrow \sigma^{-n} C \stackrel{[1]}{\longrightarrow} \text {. }
$$

Since $\sigma^{-n} C \in D^{\sigma, \leq-1}, \sigma^{-n} \phi$ is an epimorphism in $\mathcal{H}^{\sigma}$.

(2) Let

$$
M \stackrel{f}{\longrightarrow} N \longrightarrow L \stackrel{[1]}{\longrightarrow}
$$

be an exact triangle such that $M, N \in \mathcal{H}^{\sigma}$ and $L \in D^{\sigma, \leq-1}$. Take an integer $n_{0}$ such that $\operatorname{Hom}\left(A, \sigma^{n} L^{\cdot}\right) \cong \mathrm{H}^{0}\left(\sigma^{n} L^{\cdot}\right)=0$ for each $n \geq n_{0}$. Then the induced morphism $\operatorname{Hom}\left(A, \sigma^{n} M\right) \longrightarrow$ $\operatorname{Hom}\left(A, \sigma^{n} N\right)$ is surjective in $\operatorname{Mod}-A$ for each $n \geq n_{0}$.

Definition 2.6. Let $A$ be a finite dimensional $k$-algebra and let $\sigma$ be a two-sided tilting complex over A. $\sigma$ is called extremely ample if $\sigma^{n}$ is pure for $n \geq 0 . \sigma$ is called very ample if $\mathrm{H}^{i}(\sigma)=0$ for $i \geq 1$ and $\sigma^{n}$ is pure for $n \gg 0 . \sigma$ is called ample if $\sigma^{n}$ is pure for $n \gg 0$.

Let $\sigma$ be a very ample two-sided tilting complex over a finite dimensional $k$-algebra $A$. Then $\mathrm{H}^{0}(\sigma)^{\otimes_{A} n} \simeq \mathrm{H}^{0}\left(\sigma^{n}\right)$ for $n \geq 0$ by Lemma 2.4. Therefore the tensor algebra

$$
T_{A}\left(\mathrm{H}^{0}(\sigma)\right)=\bigoplus_{n \geq 0}\left(\mathrm{H}^{0}(\sigma)\right)^{\otimes_{A} n}
$$


of $\mathrm{H}^{0}(\sigma)$ over $A$ is naturally isomorphic to the homogeneous coordinate ring

$$
\Gamma_{*}\left(\mathcal{H}^{\sigma}, A, \sigma\right)=\bigoplus_{n \geq 0} \operatorname{Hom}\left(A, \sigma^{n} A\right) \cong \bigoplus_{n \geq 0} \mathrm{H}^{0}\left(\sigma^{n}\right)
$$

of the triple $\left(\mathcal{H}^{\sigma}, A, \sigma\right)$. By Theorem 1.2 we obtain the following Corollary.

Corollary 2.7. Let $A$ be a finite dimensional k-algebra of finite global dimension and let $\sigma$ be a very ample two-sided tilting complex over $A$. Then the tensor algebra $T:=T_{A}\left(\mathrm{H}^{0}(\sigma)\right)$ of $\mathrm{H}^{0}(\sigma)$ over $A$ is a graded coherent ring and the triple $\left(\mathcal{H}^{\sigma}, A, \sigma\right)$ is equivalent to the triple (cohproj $\left.T, \bar{T},(1)\right)$ as triple. In particular the abelian category $\mathcal{H}^{\sigma}$ is equivalent to the abelian category cohproj $T$.

In $[\mathrm{Be}]$ Beilinson showed that $\mathbb{P}^{n}$ is derived equivalent to a finite dimensional $k$-algebra. This result has been generalized to other varieties. The next Theorem gives a partial converse.

Theorem 2.8. Let $A$ be a finite dimensional $k$-algebra of finite global dimension and let $\sigma$ be a very ample two-sided tilting complex. Then there is a natural equivalence of triangulated categories

$$
D^{b}(\bmod -A) \stackrel{\sim}{\longrightarrow} D^{b}\left(\operatorname{cohproj} T_{A}\left(\mathrm{H}^{0}(\sigma)\right)\right) .
$$

Proof. We set $T:=T_{A}\left(\mathrm{H}^{0}(\sigma)\right)$. Let $\mathcal{P}_{A}$ be the full subcategory of mod- $A$ consisting all finite projective $A$ modules. We can extend the functor

$$
\mathcal{P}_{A} \longrightarrow \operatorname{cohproj} T, \quad P \mapsto \bigoplus_{i \geq 0} \operatorname{Hom}\left(A, P \otimes_{A} \mathrm{H}^{0}\left(\sigma^{i}\right)\right)
$$

to the functor $\gamma: K^{b}\left(\mathcal{P}_{A}\right) \longrightarrow K^{b}(\operatorname{cohproj} T)$ between the homotopy category of complexes. Let $\Phi: K^{b}\left(\mathcal{P}_{A}\right) \stackrel{\sim}{\longrightarrow} D^{b}(\bmod -A)$ be the natural equivalence and let $\pi: K^{b}(\operatorname{cohproj} T) \longrightarrow D^{b}(\operatorname{cohproj} T)$ be the natural quotient functor. Define $\mathbf{L} \bar{\Gamma}_{*}:=\pi \circ \gamma \circ \Phi^{-1}$. Then we obtain the following commutative diagram.

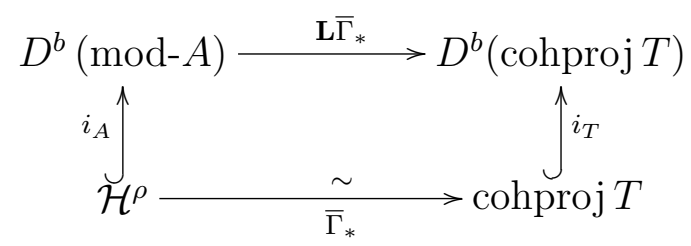

where $i_{A}, i_{\Pi}$ are inclusions. We prove that $\mathbf{L} \bar{\Gamma}_{*}$ is an equivalence.

By Lemma 2.5 and Theorem 1.2 the functor $\bar{\Gamma}_{*}: \mathcal{H}^{\sigma} \longrightarrow \operatorname{cohproj} T$ is an equivalence. Therefore $\mathbf{L} \bar{\Gamma}_{*}$ is essentially surjective. To complete the proof it suffices to show that $\mathbf{L} \bar{\Gamma}_{*}$ is fully faithful. Since every complex $M^{\cdot} \in D^{b}(\bmod -A)$ is obtained from $A$ by taking finite number of cones, shifts and direct summand, the problem is reduced to the following lemma.

Lemma 2.9. The map

$$
\operatorname{Hom}_{D^{b}(\bmod -A)}(A, A[i]) \stackrel{\mathbf{L} \bar{\Gamma}_{* A, A[i]}}{\longrightarrow} \operatorname{Hom}_{D^{b}(\operatorname{cohproj} T)}\left(\mathbf{L} \bar{\Gamma}_{*}(A), \mathbf{L} \bar{\Gamma}_{*}(A)[i]\right)
$$

is an isomorphism for every $i \in \mathbb{Z}$.

Proof. For the case $i=0$, the map $\mathbf{L} \bar{\Gamma}_{* A, A}$ is equal to

$$
\begin{aligned}
\operatorname{Hom}_{D^{b}(\bmod -A)}(A, A) \cong \operatorname{Hom}_{\mathcal{H}^{\sigma}}(A, A) & \cong \operatorname{Hom}_{\operatorname{cohproj} T}\left(\bar{\Gamma}_{*}(A), \bar{\Gamma}_{*}(A)\right) \\
& \cong \operatorname{Hom}_{D^{b}(\operatorname{cohproj} T)}\left(\mathbf{L} \bar{\Gamma}_{*}(A), \mathbf{L} \bar{\Gamma}_{*}(A)\right)
\end{aligned}
$$


where the second isomorphism is induced by the equivalence $\bar{\Gamma}_{*}$. Hence $\mathbf{L} \bar{\Gamma}_{* A, A}$ is an isomorphism.

For the case $i \neq 0$, since $\mathbf{L} \bar{\Gamma}_{*}(A) \cong \bar{T}$, we have only to show that $\operatorname{Ext}_{q c o h T}^{i}(\bar{T}, \bar{T})=0$ for $i \geq 1$. First note that $\operatorname{Ext}_{\text {cohproj } T}^{i}(\bar{T}, \bar{T}) \cong \lim _{n \rightarrow \infty} \operatorname{Ext}_{\text {coh } T}^{i}\left(T_{\geq n}(-n), T\right)$ where $T_{\geq n}:=\oplus_{m \geq n} \mathrm{H}^{0}(\sigma)^{\otimes_{A} m}$ and $(-n)$ is the $-n$-graded degree shift operator (See [AZ]). Let $n_{0} \geq 0$ be a positive integer such that $\sigma^{m}$ is pure for $m \geq n_{0}$. Then by Lemma $2.4 \mathrm{H}^{0}(\sigma)^{\otimes_{A} m+n} \simeq \mathrm{H}^{0}(\sigma)^{\otimes_{A} m} \otimes_{A}^{\mathbf{L}} \sigma^{n}$ for $n \geq n_{0}$ and $m \geq 0$. Thus $T_{\geq n} \simeq T \otimes_{A}^{\mathbf{L}} \sigma^{n}$ for $n \geq n_{0}$. Therefore for $n \geq n_{0}$

$$
\begin{aligned}
\mathbb{R} \operatorname{Hom}_{\operatorname{coh} T}\left(T_{\geq n}(-n), T\right) \simeq \mathbb{R} \operatorname{Hom}_{\operatorname{coh} T}\left(T \otimes_{A}^{\mathbf{L}} \sigma^{n}(-n), T\right) & \simeq \mathbb{R} \operatorname{Hom}_{\operatorname{coh} T}\left(T, \sigma^{-n} \otimes_{A}^{\mathbf{L}} T(n)\right) \\
& \simeq \sigma^{-n} \otimes_{A}^{\mathbf{L}} \sigma^{n} \simeq A .
\end{aligned}
$$

This complete the proof of the Lemma, which also complete the proof of Theorem 2.8.

Lemma 2.10. Let $A$ be a finite dimensional $k$-algebra of finite global dimension and let $\sigma$ be a very ample two-sided tilting complex over $A$. Then $D^{\sigma}=D^{\sigma^{n}}$ for $n \in \mathbb{N}$.

Definition 2.11. Let $A$ be a finite dimensional $k$-algebra of finite global dimension and let $\sigma$ be an ample two-sided tilting complex over $A$. We define $D^{\sigma}:=D^{\sigma^{n}}$ and $\mathcal{H}^{\sigma}:=\mathcal{H}^{\sigma^{n}}$ where $n$ is a natural number such that $\sigma^{n}$ is very ample. By the above Lemma, this is well-defined.

Proposition 2.12. Let $A$ be a finite dimensional $k$-algebra of finite global dimension and let $\sigma$ be an ample two-sided tilting complex. Then the following conditions are equivalent.

(1) the inverse $\sigma^{-1}$ is ample.

(2) the $t$-structure $D^{\sigma}$ is equal to the standard $t$-structure $D^{A}$.

(3) $\sigma^{n} \in \operatorname{Pic} A$ for $n \gg 0$.

Lemma 2.13. If $M \in \mathcal{H}^{\sigma}$, then $\mathrm{H}^{i}(M)=0$ for $i<0$ and $i>\max \left\{\operatorname{pd}\left({ }_{A} \sigma^{n}\right) \mid n \geq 0\right\}$ where $\operatorname{pd}\left({ }_{A} \sigma^{n}\right)$ is the projective dimension of $\sigma^{n}$ as a left $A$-module.

Proof. We prove that $\mathrm{H}^{i}(M)=0$ for $i>\max \left\{\operatorname{pd} \sigma^{n} \mid n \geq 0\right\}$. The case when $i<0$ can be proved in the same way. Set $d:=\max \left\{\operatorname{pd}\left({ }_{A} \sigma^{n}\right) \mid n \geq 0\right\}$. Let $\tau_{\leq d}$ and $\tau_{\geq d+1}$ be the standard truncation functors of $D^{b}(\bmod -A)$. We have the following exact triangle:

$$
\sigma^{n}\left(\tau_{\leq d} M\right) \longrightarrow \sigma^{n} M \longrightarrow \sigma^{n}\left(\tau_{\geq d+1} M\right) \stackrel{[1]}{\longrightarrow}
$$

for each $n \geq 0$. Let $n$ be a positive integer such that $\sigma^{n} M$ and $\sigma^{n}$ are pure. Since $d=\max \left\{\operatorname{pd}\left({ }_{A} \sigma^{n}\right) \mid\right.$ $n \geq 0\}, \mathrm{H}^{i}\left(\sigma^{n}\left(\tau_{\geq d+1} M\right)\right)=0$ for $i \leq 0$. For $i \geq 1$, we consider the following part of the cohomology long exact sequence

$$
\mathrm{H}^{i}\left(\sigma^{n} M\right)=0 \longrightarrow \mathrm{H}^{i}\left(\sigma^{n}\left(\tau_{\geq d+1} M\right)\right) \stackrel{\partial^{i}}{\longrightarrow} \mathrm{H}^{i+1}\left(\sigma^{n}\left(\tau_{\leq d} M\right)\right)
$$

of the exact triangle (4). Applying the same argument in the proof of Theorem 2.2, we conclude that the connecting morphism $\partial^{i}=0$ and $\mathrm{H}^{i}\left(\sigma^{n}\left(\tau_{\geq d+1} M\right)\right)=0$ for $i \geq 1$. Therefore $\sigma^{n}\left(\tau_{\geq d+1} M\right)=0$ and hence $\tau_{\geq d+1} M=0$. This completes the proof.

Let $\mathcal{P}=(\mathcal{C}, \mathcal{O})$ be a pair consisting of a $k$-linear abelian category $\mathcal{C}$ and an object $\mathcal{O} \in \mathcal{C}$. In [AZ, Sction 7] the cohomology group of $\mathcal{F} \in \mathcal{C}$ is defined to be $H^{i}(\mathcal{P}, \mathcal{F}):=\operatorname{Ext}_{\mathcal{C}}^{i}(\mathcal{O}, \mathcal{F})$. for $i \geq 0$. The cohomological dimension of the pair $\mathcal{P}$ is defined to be

$$
\operatorname{cd}(\mathcal{P}):=\max \left\{i \mid \mathrm{H}^{i}(\mathcal{P}, \mathcal{F}) \neq 0 \text { for } \mathcal{F} \in \mathcal{C}\right\}
$$

By definition cohomology groups and cohomological dimension of a triple $(\mathcal{C}, \mathcal{O}, s)$ in Section 1 are that of the pair $(\mathcal{C}, \mathcal{O})$.

Let $A$ be a finite dimensional $k$-algebra of finite global dimension and let $\sigma$ be a very ample tilting complex over $A$. Set $T=T_{A}\left(\mathrm{H}^{0}(\sigma)\right)$. We consider the triple $\operatorname{proj} T=(\operatorname{cohproj} T, \bar{T},(1))$. 
Corollary 2.14. $\operatorname{cd}(\operatorname{proj} T)=\max \left\{\operatorname{pd}\left({ }_{A} \sigma^{n}\right) \mid n \geq 0\right\}$.

Proof. Let $\mathcal{F} \in \operatorname{cohproj} T$ and let $M \in \mathcal{H}^{\sigma}$ be an object of $D^{b}(\bmod -A)$ which corresponds to $\mathcal{F}$ under the equivalence of Theorem 2.8. Then $\mathrm{H}^{i}(\operatorname{proj} T, \mathcal{F}) \cong \operatorname{Hom}(A, M[i]) \cong \mathrm{H}^{i}(M)$ where the right hand side is the $i$-th cohomology group of the complex $M$ of right $A$-modules. Therefore by Lemma 2.13 $\mathrm{H}^{i}(\operatorname{proj} T, \mathcal{F})=0$ for $i<0, \max \left\{\operatorname{pd} \sigma^{n} \mid n \geq 0\right\}<i$. Hence $\operatorname{cd}(\operatorname{proj} T) \leq \max \left\{\operatorname{pd} \sigma^{n} \mid n \geq 0\right\}$. It is clear that $\sigma^{-n} A \in \mathcal{H}^{\sigma}$. If $\operatorname{pd}\left({ }_{A} \sigma^{n}\right)=d$, then $\mathrm{H}^{d}\left(\sigma^{-n} A\right) \neq 0$. Therefore $\operatorname{cd}(\operatorname{proj} T)=\max \left\{\operatorname{pd} \sigma^{n} \mid\right.$ $n \geq 0\}$.

The global dimension of cohproj $T$ is bounded by gl. $\operatorname{dim} A$ from above.

Proposition 2.15. gl. $\operatorname{dim}(\operatorname{cohproj} T) \leq$ gl. $\operatorname{dim} A$.

Proof. Let $\mathcal{F}, \mathcal{G} \in \operatorname{cohproj} T$ and let $M, N \in \mathcal{H}^{\sigma}$ be an object of $D^{b}(\bmod -A)$ which corresponds to $\mathcal{F}, \mathcal{G}$ under the equivalence of Theorem 2.8. Let $n \gg 0$ be a positive integer such that $\sigma^{n} M$ and $\sigma^{n} N$ are pure. Then $\operatorname{Ext}_{\text {cohproj } T}^{i}(\mathcal{F}, \mathcal{G}) \cong \operatorname{Ext}_{\text {mod- } A}^{i}\left(\sigma^{n} M, \sigma^{n} N\right)=0$ for $i>$ gl. $\operatorname{dim} A$.

Remark 2.16. In general gl. $\operatorname{dim}(\operatorname{cohproj} T)<\operatorname{gl} . \operatorname{dim} A$. See Section 3.3.

\section{Fano algebras and algebras with ample canonical bundle}

\section{1 definition and basic properties}

Let $A$ be a finite dimensional $k$-algebra of finite global dimension. The $k$-dual $A^{*}$ has the natural $A$-bimodule structure. It is known that $-\otimes_{A}^{\mathbf{L}} A^{*}: D^{b}(\bmod -A) \longrightarrow D^{b}(\bmod -A)$ is the Serre functor ([Hap, I.4.6]). For a nonsingular projective variety $X$ over $k$, the $[\operatorname{dim} X]$-shifted derived tensor $-\otimes_{X}^{\mathbf{L}} \omega_{X}[\operatorname{dim} X]$ of the canonical bundle $\omega_{X}$ is the Serre functor of $D^{b}(\operatorname{coh} X)$. From a view point of noncommutative algebraic geometry $A^{*}$ is thought as "shifted canonical bundle". For example, if $\left(A^{*}\right)^{m} \simeq[n]$ for some positive integers $m, n$, then $A$ is called fractional Calabi-Yau of $C Y$ dimension $\frac{n}{m}$, which is apparently named after analogy to the property of the derived category of a Calabi-Yau variety.

Definition 3.1. Let $A$ be a finite dimensional $k$-algebra of finite global dimension, let $d$ be a nonnegative integer, and set $\omega:=\left(A^{*}[-d]\right)$. A is said to be a Fano algebra of Fano dimension $d$ if the two-sided tilting complex $\omega^{-1}$ is ample.

Remark 3.2. It is not known that in general finite dimensional k-algebras of finite global dimension have ample $A^{*}[-d]$ for some $d$. Therefore we don't use the term "algebra of general type".

Remark 3.3. (1) Let $A$ be a finite dimensional k-algebra of finite global dimension. If for some positive integer $d, \operatorname{Ext}^{i}\left(A^{*}, A\right)=0, i \neq d$, then gl. $\operatorname{dim} A=d$. Therefore if $\omega_{A}^{-1}=\left(A^{*}[-d]\right)^{-1}$ is extremely ample, then the global dimension of $A$ is equal to $d$. In general global dimension of a Fano algebra is not equal to its Fano dimension. (See Section3.3.)

(2) By the standard argument we can prove that if $\omega:=A^{*}[-d]$ is ample (resp. anti-ample) then gl. $\operatorname{dim} \mathcal{H}^{\omega}=d$ (resp. gl. $\operatorname{dim} \mathcal{H}^{\omega^{-1}}=d$ ).

Lemma 3.4. Let $A$ be a finite dimensional k-algebra of finite global dimension. If $A$ is fractional Calabi-Yau then $A$ is not Fano. Conversely if $A$ is Fano then $A$ is not fractional Calabi-Yau.

Proof. We prove that a fractionally Calabi-Yau algebra is not Fano. We set $\omega:=A^{*}[-d]$ for some $d \geq 0$. Let $m, n$ be integers such that $\left(A^{*}\right)^{m} \simeq[n]$. Then $\omega^{-m} \simeq[d m-n]$. If $d m-n \neq 0$, then $\omega^{-l m}$ is not pure for $l>0$. If $d m-n=0$ then $\omega^{-(l m-1)} \simeq \omega=A^{*}[-d]$ is not pure for $l>0$. In any case $\omega^{-1}$ is not ample. 
Example 3.5 (Geometric algebras). Let $\mathcal{T}$ be an algebraic $k$-linear triangulated category such that $\operatorname{dim}_{k} \operatorname{Hom}(E, F)<\infty$ for $E, F \in \mathcal{T}$ and $E_{\bullet}:=\left(E_{0}, E_{1}, \ldots, E_{d}\right)$ be a full geometric collection in $\mathcal{T}$ (See [BP, ELO] for the definition and the properties below of a geometric collection). The endomorphism algebra $A:=$ End $\left(\oplus_{i=0}^{d} E_{i}\right)$ is called a geometric algebra in [BP]. Then $A$ is a finite dimensional $k$-algebra of global dimension d and $\left(A^{*}[-d]\right)^{-n}$ is pure for $n \geq 0$. Therefore the geometric algebra $A$ is a Fano algebra. By Corollary 2.7, The tensor algebra $T_{A}(\rho)$ is coherent. Therefore the $\mathbb{Z}$-algebra $\mathcal{A}$ associated to geometric collection $E_{\bullet}$ is coherent. In particular, the homogeneous coordinate ring $\mathcal{A}^{m, V}$ of noncommutative Grassmanian $\operatorname{NGr}(m, V)$ ([ELO]) is coherent.

\subsection{A noncommutative algebro-geometric characterization of represen- tation type of a quiver}

Let $Q$ be a finite acyclic quiver, i.e., a quiver with finitely many vertexes and finitely many arrows without loops and oriented cycles. Then the path algebra $A=k Q$ of $Q$ is a finite dimensional $k$-algebra of global dimension 1 . Note that $\omega_{Q}^{-1}=\left(A^{*}[-1]\right)^{-1}$ is the inverse of the Auslander-Reiten translation. Therefor if the quiver $Q$ has infinite representation type, then $\omega_{Q}^{-n}$ is pure for any $n \geq 0$ by [Hap, II.4.7]. Therefore the anti-canonical bundle $\omega_{Q}^{-1}$ is extremely ample.

Theorem 3.6. Let $Q$ be a finite acyclic quiver of infinite representation type. Then the path algebra $k Q$ of $Q$ is a Fano algebra of Fano dimension 1.

If a finite acyclic quiver $Q$ has finite representation type, then its path algebra $k Q$ is fractional Calabi-Yau. (This fact has been known by specialists. See [MY] for the precise CY dimension of these algebras.) By Lemma 3.4 and Theorem 3.6 we obtain the following characterization of representation type of a quiver from a noncommutative algebro-geometric view point.

Corollary 3.7. A finite acyclic quiver has finite representation type if and only if its path algebra is fractional Calabi-Yau, and a finite acyclic quiver has infinite representation type if and only if its path algebra is Fano.

By Theorem 2.8 and Theorem 3.6 we obtain the following corollary.

Corollary 3.8. Let $Q$ be a finite acyclic quiver of infinite representation type. Then there is a natural equivalence of triangulated categories

$$
D^{b}(\bmod -k Q) \stackrel{\sim}{\longrightarrow} D^{b}(\operatorname{cohproj} \Pi(Q))
$$

where $\Pi(Q)$ is the preprojective algebra of $Q$.

Remark 3.9. The above equivalence is essentially proved in [Le].

Remark 3.10. Set $\mathcal{T}^{\prime}=\left\{N \in \bmod -A \mid \operatorname{Hom}\left(A, \omega_{Q}^{-n} N\right)=0\right.$ for $\left.n \gg 0\right\}$ and $\mathcal{F}^{\prime}=\{N \in \bmod -A \mid$ $\operatorname{Ext}^{-1}\left(A, \omega_{Q}^{-n} N\right)=0$ for $\left.n \gg 0\right\}$. Then we can prove that $\left(\mathcal{T}^{\prime}, \mathcal{F}^{\prime}\right)$ is a torsion pair on mod- $A$. From this torsion pair we can define a t-structure in $D^{b}(\bmod -A)$ by setting

$$
\begin{aligned}
& D^{\prime \geq 0}:=\left\{M^{\cdot} \in D^{\geq 0}(\bmod -A) \mid H^{0}\left(M^{\cdot}\right) \in \mathcal{F}^{\prime}\right\} \\
& D^{\prime \leq 0}:=\left\{M \in D^{\leq 1}(\bmod -A) \mid H^{1}\left(M^{\cdot}\right) \in \mathcal{T}^{\prime}\right\} .
\end{aligned}
$$

(See [HRS, Proposition I.2.1]). However, this is not a new t-structure. It can be proved that $\left(D^{\prime \geq 0}, D^{\prime \leq 0}\right)=D^{\omega_{Q}^{-1}}$. 
Remark 3.11. Let $Q$ be a finite acyclic quiver. By Happel's theorem ([Hap, Theorem.II.4.9]), there is a natural equivalence of triangulated categories

$$
D^{b}(\bmod -k Q) \stackrel{\sim}{\longrightarrow} \operatorname{grmod}-T(Q)
$$

where $T(Q):=k Q \oplus(k Q)^{*}$ is a trivial extension algebra and grmod-T $\left.Q\right)$ is the stable category of finite graded $T(Q)$ modules. In the case $Q$ has infinite representation type, compositing above equivalence and the equivalence of corollary 3.8, we obtain the equivalence of triangulated categories

$$
D^{b}(\operatorname{cohproj} \Pi(Q)) \simeq \underline{\operatorname{grmod}}-T(Q) .
$$

It seems that this equivalence asserts that $\Pi(Q)$ and $T(Q)$ are Koszul dual to each other over $k Q$. In the classical theory of Koszul algebras, graded algebras over a semi-simple algebra are treated. But path algebras are not semi-simple in general. The related theory will be developed in [MT].

\section{3 canonical algebras}

The concept of a weighted projective line was given by Geigle and Lewnzing [GL] to treat geometrically canonical algebras.

Let $p=\left(p_{0}, \ldots, p_{n}\right)$ be the $n+1$-tuple of positive integers, called a weight sequence. Denote by $\mathbf{L}(\mathbf{p})$ the rank one abelian group on generators $\vec{x}_{0}, \ldots, \vec{x}_{n}$ with relations $p_{0} \vec{x}_{0}=\cdots=p_{n} \vec{x}_{n}$. The element $\vec{c}=p_{0} \vec{x}_{0}=\cdots=p_{n} \vec{x}_{n}$ is called the canonical element of $\mathbf{L}(p)$ and the element $\vec{\omega}=(n-1) \vec{c}-$ $\sum_{i=0}^{n} \vec{x}_{i}$ is called the dualizing element of $\mathbf{L}(p) . \mathbf{L}(p)$ is an ordered group with $\mathbf{L}(p)^{+}=\sum_{i=0}^{n} \mathbb{N} \vec{x}_{i}$ as its set of positive elements.

Let $\mathbb{X}=\mathbb{X}(p, \lambda)$ be a weighted projective line of type $p=\left(p_{0}, \ldots, p_{n}\right)$ and $\lambda=\left(\lambda_{2}, \ldots, \lambda_{n}\right)$ where $\lambda$ is a sequence of pairwise distinct elements of $k^{\times}$, normalized such that $\lambda_{2}=1$.

The abelian category coh $\mathbb{X}$ of coherent sheaves on $\mathbb{X}$ has global dimension 1 .

For each $\vec{x} \in \mathbf{L}(p)$ we can attach a line bundle $\mathcal{O}_{\mathbb{X}}(\vec{x})$. This correspondence is additive, i.e., there are a natural isomorphisms $\mathcal{O}_{\mathbb{X}}(\vec{x}+\vec{y}) \cong \mathcal{O}_{\mathbb{X}}(\vec{x}) \otimes_{\mathbb{X}} \mathcal{O}_{\mathbb{X}}(\vec{y})$ and $\mathcal{O}_{\mathbb{X}}(0) \cong \mathcal{O}_{\mathbb{X}}$.

- (Serre duality) The functor $-\otimes_{\mathbb{X}}^{\mathbf{L}} \mathcal{O}_{\mathbb{X}}(\vec{\omega})[1]$ is the Serre functor of $D^{b}(\operatorname{coh} \mathbb{X})$.

- (Serre vanishing) Let $\vec{x} \in \mathbf{L}(p)^{+}$. For $\mathcal{F} \in \operatorname{coh} \mathbb{X}$,

$$
\mathrm{H}^{i}\left(\mathbb{X}, \mathcal{F} \otimes_{\mathbb{X}} \mathcal{O}_{\mathbb{X}}(n \vec{x})\right)=\operatorname{Ext}_{\operatorname{coh} \mathbb{X}}^{i}\left(\mathcal{O}_{\mathbb{X}}, \mathcal{F} \otimes_{\mathbb{X}} \mathcal{O}_{\mathbb{X}}(n \vec{x})\right)=0
$$

for $i>0$ and $n \gg 0$.

The endomorphism algebra $\Lambda=\operatorname{End}(T)$ of $T:=\bigoplus_{0 \leq \vec{x} \leq \vec{c}} \mathcal{O}(\vec{x})$ is isomorphic to a canonical algebra in the sense of Ringel $[R]$. It is given by the quiver

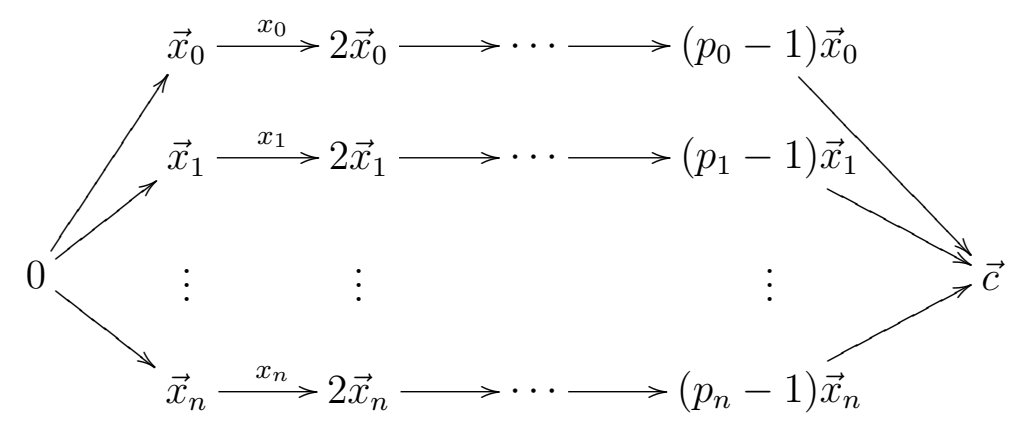

with relations $x_{i}^{p_{i}}-x_{1}^{p_{1}}+\lambda x_{0}^{p_{0}}, i=2, \ldots, n$. The global dimension of the canonical algebra $\Lambda$ is bounded by 2 from above. Moreover $T$ is a tilting sheaf on $\mathbb{X}$, i.e., $T$ induces a natural equivalence of triangulated categories

$$
D^{b}(\operatorname{coh} \mathbb{X}) \simeq D^{b}(\bmod -\Lambda)
$$


The genus $g_{\mathbb{X}}$ of a weighted projective line $\mathbb{X}$ is by definition $g_{\mathbb{X}}=1+\frac{1}{2}\left((n-1)-\sum_{i=0}^{n} \frac{p}{p_{i}}\right)$. If $g_{\mathbb{X}}<1\left(g_{\mathbb{X}}=1\right.$ resp. $\left.g_{\mathbb{X}}>1\right)$, then $\mathbb{X}$ is called of domestic (tubular resp. wild) type. Note that if $g_{\mathbb{X}}<1\left(g_{\mathbb{X}}=1\right.$ resp. $\left.g_{\mathbb{X}}>1\right)$, then $\vec{\omega}<0(\vec{\omega}=0$ resp. $\vec{\omega}>0)$.

Set $\omega_{\Lambda}:=\Lambda^{*}[-1]$. Let $\mathcal{F}, \mathcal{G} \in D^{b}(\operatorname{coh} \mathbb{X})$ and let $M, N \in \mathcal{H}^{\sigma}$ be an object of $D^{b}(\bmod -\Lambda)$ which corresponds to $\mathcal{F}, \mathcal{G}$ under the equivalence (5). Then by the uniqueness of Serre functor there is a natural isomorphism

$$
\operatorname{Hom}_{D^{b}(\operatorname{coh} \mathbb{X})}\left(\mathcal{F}, \mathcal{G} \otimes_{\mathbb{X}}^{\mathbf{L}} \mathcal{O}_{\mathbb{X}}(n \vec{\omega})\right) \cong \operatorname{Hom}_{D^{b}(\bmod -\Lambda)}\left(M, N \otimes_{\Lambda}^{\mathbf{L}} \omega_{\Lambda}^{n}\right)
$$

for $n \in \mathbb{Z}$.

In the domestic case, by Serre vanishing theorem we can prove that the canonical algebra $\Lambda$ is a Fano algebra of Fano dimension 1. The triple $\left(\mathcal{H}^{\omega_{\Lambda}^{-1}}, \Lambda, \omega_{\Lambda}^{-1}\right)$ is equivalent to $\left(\operatorname{coh} \mathbb{X}, T,-\otimes_{\mathbb{X}}^{\mathbf{L}} \mathcal{O}(-\vec{\omega})\right)$ under the equivalence (5) as a triple.

In the wild case, the canonical bundle $\omega_{\Lambda}=\Lambda^{*}[-1]$ is ample. The triple $\left(\mathcal{H}^{\omega_{\Lambda}}, \Lambda, \omega_{\Lambda}\right)$ is equivalent to $\left(\operatorname{coh} \mathbb{X}, T,-\otimes_{\mathbb{X}}^{\mathbf{L}} \mathcal{O}(\vec{\omega})\right)$ under the equivalence (5) as a triple. In general the global dimension gl. $\operatorname{dim} \Lambda$ of a canonical algebra $\Lambda$ is equal to 2 . In both case, equal sign is not true in the inequality of Proposition 2.15.

\author{
Department of Mathematics, \\ Kyoto University, KYoto 606-8502, \\ JAPAN \\ e-mail: minamoto@math.kyoto-u.ac.jp
}

\title{
References
}

[AZ] M. Artin, and J.J. Zhang, Noncommutative projective schemes, Adv. Math. 109 (1994), pp. $228-287$.

[Be] A. A. Beilinson, Coherent sheaves on $\mathbb{P}^{n}$ and problems of linear algebras, Func. Anal. Appl. 12 (1978),pp. 214-216.

[BP] A. I. Bondal, and A. E. Polishchuk, Homological properties of associative algebras: the method of helices, Russian Acad. Sci. Izv. Math. 42 (1994), no. 2, pp. 219-260.

[ELO] A. I. Efimov, V. A. Lunts and D. O. Orlov, Deformation theory of objects in homotopy and derived categories III: abelian categories, math.AG 0702840.

[GL] W. Geigle and H. Lenzing. A class of weighted projective curves arising in representation theory of finite dimensional algebras. In: Singularities, representation of algebras, and vector bundles. LNM 1273(1987) pp. 265-297.

[GM] S. I. Gelfand, and Yu. I. Manin, Methods of Homological Algebras, (Second edition) Springer Monographs in Mathematica. Springer-Verlag, Berlin,2003.

[Hap] D. Happel, Triangulated Categories in the Representation Theory of Finite-Dimensional Algebras, London Math. Soc. Lecture Notes 119, University Press, Cambridge, 1987.

[HRS] D. Happel, I. Reiten, and S. Smalo, Tilting in abelian categories and quasitilted algebras, Memoirs of the AMS, vol.575, Amer. Math. Soc., 1996.

[Har] R. Hartshorne, Algebraic Geometry, Graduated Texts in Mathematics, Springer-Verlag, 1977. 
[Le] H. Lenzing, Curve singularities arising from the representation theory of tame hereditary algebras, Representation theory I (Ottawa,Ont.,1984), pp. 199-231, LNM.,1177, Springer,Berlin, 1986

[ML] S. Mac Lane, Homology, Springer, Berlin, 1975.

[Mi] H. Minamoto, A noncommutative version of Beilinson's theorem, J. Alg. 320 (2008), pp. $238-252$.

[MT] H. Minamoto, and A. Takahashi, Generalized Koszul duality and its applications, in preparation.

[MY] J. Miyachi, and A. Yekutieli, Derived Picard groups of finite dimensional hereditary algebras, Compositio Math. 129 (2001), no. 3, pp. 341-368.

[Po] A. Polishchuk, Nonncommutative proj and coherent algebras, Math. Res. Lett., 12 (2005),1, pp. 63-74.

[R] C. M. Ringel. Tame Algebras and Integral Quadratic Forms. LNM. 1099 (1984).

[Y] A. Yekutieli, Dualizing complexes, Morita equivalence and the derived Picard groupof a ring, J. London Math Soc.60 (1999), pp. 723-746. 